\title{
Prostate-Specific Membrane Antigen PET/CT: False-Positive Results due to Sarcoidosis?
}

\author{
Robert M. Hermann ${ }^{a, b} \quad$ Manoutschehr Djannatian $^{a} \quad$ Norbert Czech $^{c}$ \\ Mirko Nitsche ${ }^{a, d}$ \\ ${ }^{a}$ Center for Radiotherapy and Radiooncology Bremen and Westerstede, \\ Westerstede, Germany; ${ }^{b}$ Institute for Radiation Oncology and Special Oncology, Hannover \\ Medical School, Hannover, Germany; ${ }^{C}$ Center for Nuclear Medicine and PET/CT, \\ Bremen, Germany; ${ }^{d}$ Department for Radiotherapy, Karl-Lennert Cancer Center, University \\ of Schleswig Holstein, Campus Kiel, Kiel, Germany
}

\section{Keywords}

Prostate cancer - Sarcoidosis - PET/CT · Prostate-specific membrane antigen · Radiotherapy

\begin{abstract}
We report on a 72-year-old male patient who developed sarcoidosis of the mediastinal lymph nodes, the liver, and the prostate 11 years ago. Seven years later, he underwent transurethral resection of the prostate by laser due to hematuria. Pathology of the resected chips showed a 'granulomatous prostatitis with epitheloid cells'. Malignancy was histologically excluded at that time. Four years later, he was diagnosed with an undifferentiated prostate carcinoma, with a Gleason score of $5+4=9$. After initiation of antihormonal therapy, he underwent radical prostatectomy and pelvic lymphadenectomy, which revealed a pT3b pN1 carcinoma with infiltrated resection margins. Three months later, the prostate-specific antigen level was $1.4 \mathrm{ng} / \mathrm{ml}$, and a local recurrence was suspected by ultrasound; consequently, a ${ }^{68} \mathrm{Ga}$-prostate-specific membrane antigen (PSMA) PET/CT was performed. This examination seemed to confirm the local recurrence, a right pelvic lymph node metastasis, and a hepatic metastasis. However, ultrasound with contrast medium could not confirm the metastatic spread to the liver. In palliative intention, radiotherapy of the pelvis was done. After $50 \mathrm{~Gy}$, the supposed recurrence had markedly shrunk, and an additional boost dose with $16.2 \mathrm{~Gy}$
\end{abstract}


was applied. Two years later, the patient is still free of disease. Due to this clinical development, we doubt the diagnosis of a fulminant progression of the prostate cancer as suspected by PSMA-PET/CT. Instead, we suspect a recurrence of the previously proven sarcoidosis leading to false-positive results. Our focus in this report is on the interaction between PSMA$\mathrm{PET} / \mathrm{CT}$ and sarcoidosis. Another report on a case of sarcoidosis of the spleen seems to confirm this possibility [Kobe et al: Clin Nucl Med 2015;40:897-898].

(C) 2016 The Author(s)

Published by S. Karger AG, Basel

\section{Clinical Presentation}

A 72-year-old man was referred to our Department of Radiation Oncology. The patient had been suffering from arterial hypertension. Other pre-existing disorders were psoriasis and arthritis, and the medication he took comprised antihypertensive medication. No allergies were known. He did not smoke, and alcohol consumption was denied.

Eleven years before his first presentation at our department, he was diagnosed with sarcoidosis of the mediastinal lymph nodes, the liver, and even the prostate. At that time, the prostate-specific antigen (PSA) level was elevated at $9.8 \mathrm{ng} / \mathrm{ml}$. Two biopsies revealed no malignancy; however, biopsies of the liver and a mediastinal lymph node showed sarcoidosis. The differential diagnosis of tuberculosis was not supported in serology. There was no erythema nodosum at any time.

Seven years later, he suffered from hematuria. He received a transurethral resection of the prostate and laser coagulation. Pathology of the resected chips revealed 'granulomatous prostatitis with epitheloid cells'. Malignancy was histologically excluded at that time. The patient had never received intravesical bacillus Calmette-Guérin therapy at any time.

Four years later, he was diagnosed with locally advanced and undifferentiated prostate cancer. The PSA level was just $4.1 \mathrm{ng} / \mathrm{ml}$. However, due to unintended weight loss, an MRI of the abdomen and pelvis was performed, suspicious of a malignancy of the prostate. In the following, biopsies of the prostate substantiated the diagnosis. For staging, a ${ }^{68} \mathrm{Ga}-\mathrm{PSMA}-$ PET/CT was done. The examination suggested locally advanced prostate cancer and lymph node metastases in the left pelvis. In consequence of this finding, hormone withdrawal with luteinizing hormone-releasing hormone agonists was initiated.

One month later, radical prostatectomy with pelvic lymphadenectomy was performed. Pathology revealed an adenocarcinoma of the prostate pT3b pN1 (8/18), with a Gleason score of $5+4=9$. Resection margins in dorsal and right apical direction were extensively infiltrated. One month after resection, the PSA level was $0.05 \mathrm{ng} / \mathrm{ml}$, and 3 months later, it had risen to $1.4 \mathrm{ng} / \mathrm{ml}$. Transrectal ultrasonography showed signs of a local recurrence, and thus, the PSMA-PET/CT scan was repeated. In this study, a local recurrence, a lymph node metastasis in the right pelvis, and an avid area in the liver segment VIII were found (fig. 1).

\section{Investigations/Imaging Findings}

For further clarification of a potential spread of the carcinoma into the liver, an ultrasound with contrast medium was performed. However, there was no pathological area or tumor in the liver, so no biopsies could be taken. At that time, we did not further investigate the liver (e.g. with MRI), but decided to initiate palliative radiotherapy of the local recurrence and the pelvic lymphatic pathways. 


\section{Case Reports in Oncology}

\section{Treatment}

Due to the extent of the local recurrence, the radiation dose to the tumor region was initially limited to a total dose of $59.4 \mathrm{~Gy}$ (single dose $1.8 \mathrm{~Gy}$ ). In 3D planning, the pelvic lymphatics received $50.4 \mathrm{~Gy}$. An additional boost dose with 9 Gy was administered in the region of the macroscopic lymph node metastasis in the right internal iliac region. The patient tolerated radiation therapy fairly well. For a mild proctitis, he received a foam containing a steroid for rectal application.

After application of a dose of $50.4 \mathrm{~Gy}$, a restaging CT scan was done. In this scan, a reduction in the recurrence from 84 to $32 \mathrm{ml}$ was documented (fig. 2). Treatment fields were adapted to the new tumor extension, thus enabling a protection of the anterior rectal wall and an escalation of the prescribed dose to $66.6 \mathrm{~Gy}$. Therapy could be completed as scheduled without further increase in toxicity.

\section{Outcome and Follow-Up}

Three months after completion of radiotherapy, the patient's PSA level had decreased to $0.02 \mathrm{ng} / \mathrm{ml}$. No residual tumor mass could be shown in the restaging CT scan at that time. Furthermore, no hepatic anomalies or tumors could be identified in ultrasonography. Two years later, the patient was well, with a stable weight and good appetite. Proctitis had resolved, but he still suffered from significant bladder incontinence.

\section{Discussion}

The complete response to radiotherapy described above is unusual in the context of a rapidly regrowing ( 3 months after curative surgery), highly aggressive and metastasizing prostate cancer. This led us to the hypothesis that the underlying sarcoidosis of the prostate might have mimicked - at least in parts - the recurrent tumor masses and the presumed metastases.

To support the suspicion of sarcoidosis in the liver, an MRI might have been useful [1]; however, at that time, we were focusing on the exclusion of metastatic spread of the prostate cancer. As contrast-enhanced ultrasonography did not reveal any suspicious areas, we did not further investigate this organ.

Sarcoidosis is a multisystem inflammatory disease, pathologically characterized by noncaseating granulomas [2]. It can affect any organ or anatomical region of the body, predominantly the lungs and the skin [3]. The incidence is about 20/100.000 [3]. However, as immune-modulating therapies play an increasingly important role in oncology, this incidence might increase. In a previous study, 5 out of 70 patients receiving vemurafenib (a BRAF inhibitor) for melanoma developed sarcoidosis [4]. Other reports discuss an increased detection rate after treatment with tumor necrosis factor- $\alpha$ inhibitors [5].

An affection of the prostate is rare, with less than 20 cases reported in the literature so far $[6,7]$.

\section{Response of Sarcoidosis to Radiotherapy}

The prompt and complete remission of the tumor masses in the former prostate region under radiotherapy support the suspected diagnosis of sarcoidosis. 


\section{Case Reports in Oncology}

It is well known that sarcoidosis responds very well to radiotherapy, independent of the localization and prior unsuccessful treatments with steroids or other immunomodulating agents. Normal fractionated radiation doses as low as 20 Gy seem to be sufficient to treat a systemic progress with total nodal irradiation [8], while doses between 20 and 30 Gy have a curative potential in otherwise refractory neurosarcoidosis [for a review, see ref. 9]. Penile lesions have been successfully irradiated as well [10]. However, due to long-term risk, radiotherapy is not a therapeutic option in first-line therapy.

\section{PSMA and Prostate Cancer}

Although the term 'prostate-specific membrane antigen' suggests high specificity of expression in prostate tissues, this membrane-bound binuclear zinc metallopeptidase is also described as 'glutamate carboxypetidase $\mathrm{II}^{\prime}$, as ' $\mathrm{N}$-acetylated-alpha-linked acidic dipeptidase', or as 'folate hydrolase' (FOLH1) [11]. Different tissues express this protein in vivo [for an overview, see ref. 11]: secretory-acinar epithelium of the prostate, astrocytes and Schwann cells of the nervous system, proximal tubules in the kidneys and the small intestine. Far lower expressions have been found in tissues of the heart, pancreas, bladder, skin, breast, liver, lung, colon, and testis. A higher expression of PSMA has been seen on the apical and luminal surface of new blood endothelial cells in tumor neovasculature and in the neovasculature of some normal proliferative tissues (endometrium, heart valve injuries, pleural lesions, and keloid scars) [12].

In malignant cells, the highest levels of expression have been described in androgenresistant prostate cancer [11]. In this context, PSMA-PET/CT is extremely useful in the imaging of early recurrent prostate cancer. In a series of 310 patients, Afshar-Oromieh et al. [13] described a sensitivity of $76.6 \%$, a specificity of $100 \%$, a negative predictive value of $91.4 \%$, and a positive predictive value of $100 \%$ in a lesion-based analysis. Although 27 patients had PSA values $<0.5 \mathrm{ng} / \mathrm{ml}$, these excellent results were obtained.

\section{PSMA-PET/CT and Sarcoidosis}

Recently, a false-positive finding in PSMA-PET/CT in the context of sarcoidosis has been reported [14]. In a patient with prostate cancer, PSMA-positive tissue was found in the spleen with intense tracer take-up. As this localization is rather unusual for metastases of prostate cancer, a biopsy was taken. Malignancy was excluded in pathology, and a sarcoidosis confirmed.

Expression of PSMA in cells forming sarcoid lesions has yet not been reported in biochemical studies. However, there is evidence that activated macrophages (that play a major role in autoimmune diseases) express high levels of folic acid receptors [15]. Theoretically, these might interfere with the expression of 'folate hydrolase'/PSMA.

Concluding, in patients with a history of sarcoidosis, the results of a PSMA-PET/CT must be interpreted with caution. A histological confirmation of suspicious findings is highly recommended.

\section{Statement of Ethics}

The authors have no ethical conflicts to disclose. 


\section{Case Reports in Oncology}

Disclosure Statement

The authors have no conflicts of interest to declare.

\section{References}

1 Wada N, Nouso K, Kariyama K, Wakuta A, Kishida M, Nishimura M, Higashi T: Liver sarcoidosis with unique MRI images using gadolinium ethoxybenzyl diethylenetriamine pentaacetic acid. Acta Med Okayama 2015;69:307-311.

-2 Liu D, Birnbaum AD: Update on sarcoidosis. Curr Opin Ophthalmol 2015;26:512-516.

3 La Rochelle JC, Coogan CL: Urological manifestations of sarcoidosis. J Urol 2012;187:18-24

-4 Lheure C, Kramkimel N, Franck N, Laurent-Roussel S, Carlotti A, Queant A, Goldwasser F, Avril MF, Dupin N: Sarcoidosis in patients treated with vemurafenib for metastatic melanoma: a paradoxical autoimmune activation. Dermatology 2015;231:378-384.

5 Amber KT, Bloom R, Mrowietz U, Hertl M: TNF- $\alpha$ : a treatment target or cause of sarcoidosis? J Eur Acad Dermatol Venereol 2015;29:2104-2111.

-6 Mulpuru SK, Gujja K, Pai VM, Chen CY, Levey RL: A rare and unusual cause of PSA (prostate-specific antigen) elevation: sarcoidosis of the prostate. Am J Med Sci 2008;335:246.

7 Maurice MJ, Zhu H: Sarcoidosis of the prostate. J Urol 2013;190:711-712.

8 Ahmad K, Kim YH, Spitzer AR, Gupta A, Han IH, Herskovic A, Sakr WA: Total nodal radiation in progressive sarcoidosis. Case report. Am J Clin Oncol 1992;15:311-313.

-9 Motta M, Alongi F, Bolognesi A, Cozzarini C, Di Muzio N, Fazio F: Remission of refractory neurosarcoidosis treated with brain radiotherapy: a case report and a literature review. Neurologist 2008;14:120-124.

10 Whittaker M, Anderson CK, Clark PB: Sarcoidosis of the penis treated by radiotherapy. Br J Urol 1975;47:325-330.

11 Barinka C, Rojas C, Slusher B, Pomper M: Glutamate carboxypeptidase II in diagnosis and treatment of neurologic disorders and prostate cancer. Curr Med Chem 2012;19:856-870.

12 Foss CA, Mease RC, Cho SY, Kim HJ, Pomper MG: GCPII imaging and cancer. Curr Med Chem 2012;19:1346-1359.

$\$ 13$ Afshar-Oromieh A, Avtzi E, Giesel FL, Holland-Letz T, Linhart HG, Eder M, Eisenhut M, Boxler S, Hadaschik BA, Kratochwil C, Weichert W, Kopka K, Debus J, Haberkorn U: The diagnostic value of PET/CT imaging with the (68)Ga-labelled PSMA ligand HBED-CC in the diagnosis of recurrent prostate cancer. Eur J Nucl Med Mol Imaging 2015;42:197-209.

14 Kobe C, Maintz D, Fischer T, Drzezga A, Chang DH: Prostate-specific membrane antigen PET/CT in splenic sarcoidosis. Clin Nucl Med 2015;40:897-898.

15 Shen J, Chelvam V, Cresswell G, Low PS: Use of folate-conjugated imaging agents to target alternatively activated macrophages in a murine model of asthma. Mol Pharm 2013;10:1918-1927. 


\section{Case Reports in Oncology}
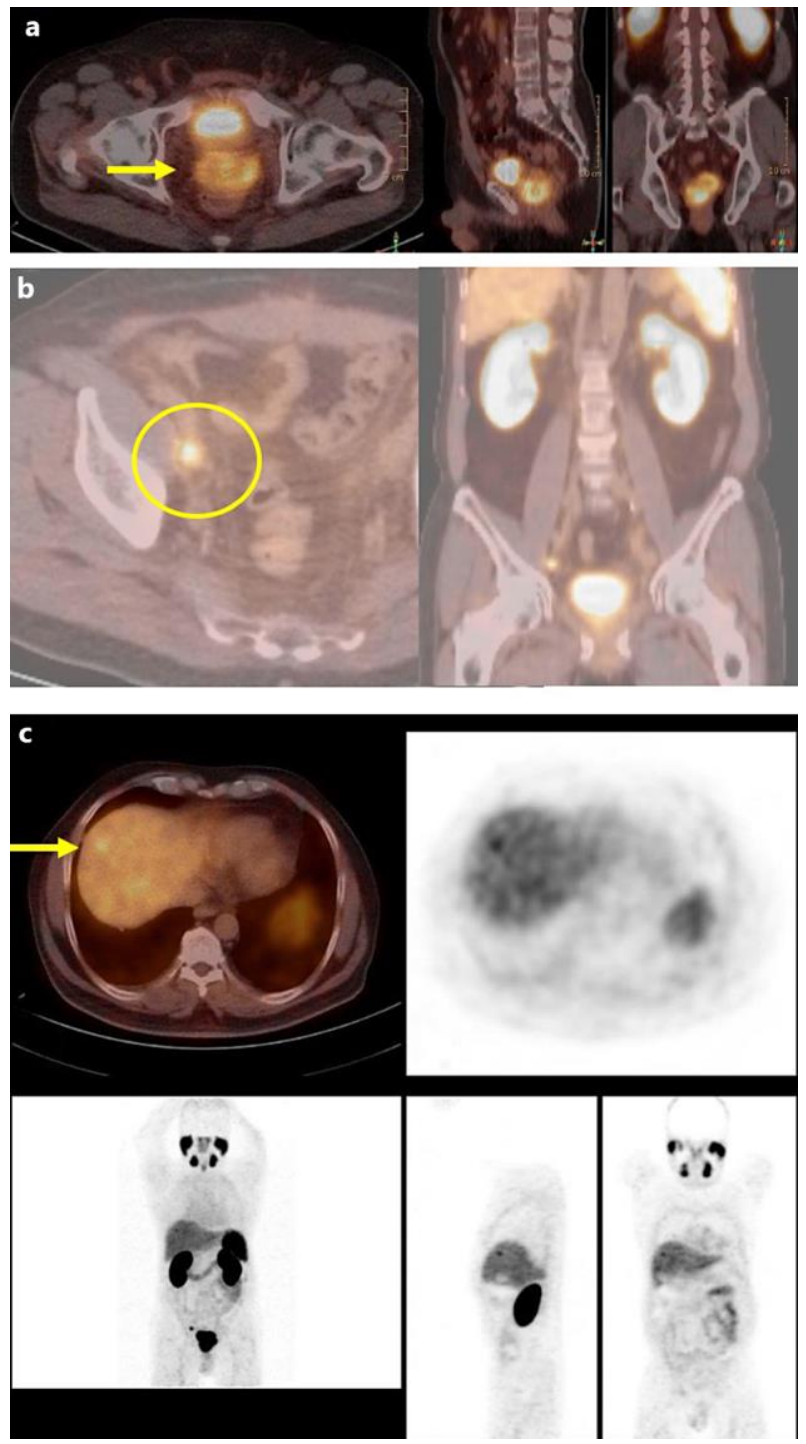

Fig. 1. Staging PSMA-PET/CT scan showing the supposed rapid prostate cancer recurrence 3 months after radical prostatectomy. In the prostatic fossa, a large PSMA-positive tumor with a SUV $\max$ up to 10.6 is found (a, arrow). Furthermore, a lymphatic metastasis in the right iliac region was detected (SUV $\max 5.4$, about 1 $\mathrm{cm}$ in diameter; $\mathbf{b}$, circle). In segment 8 of the liver, a focal PSMA take-up is shown (c, arrow). 


\section{Case Reports in Oncology}
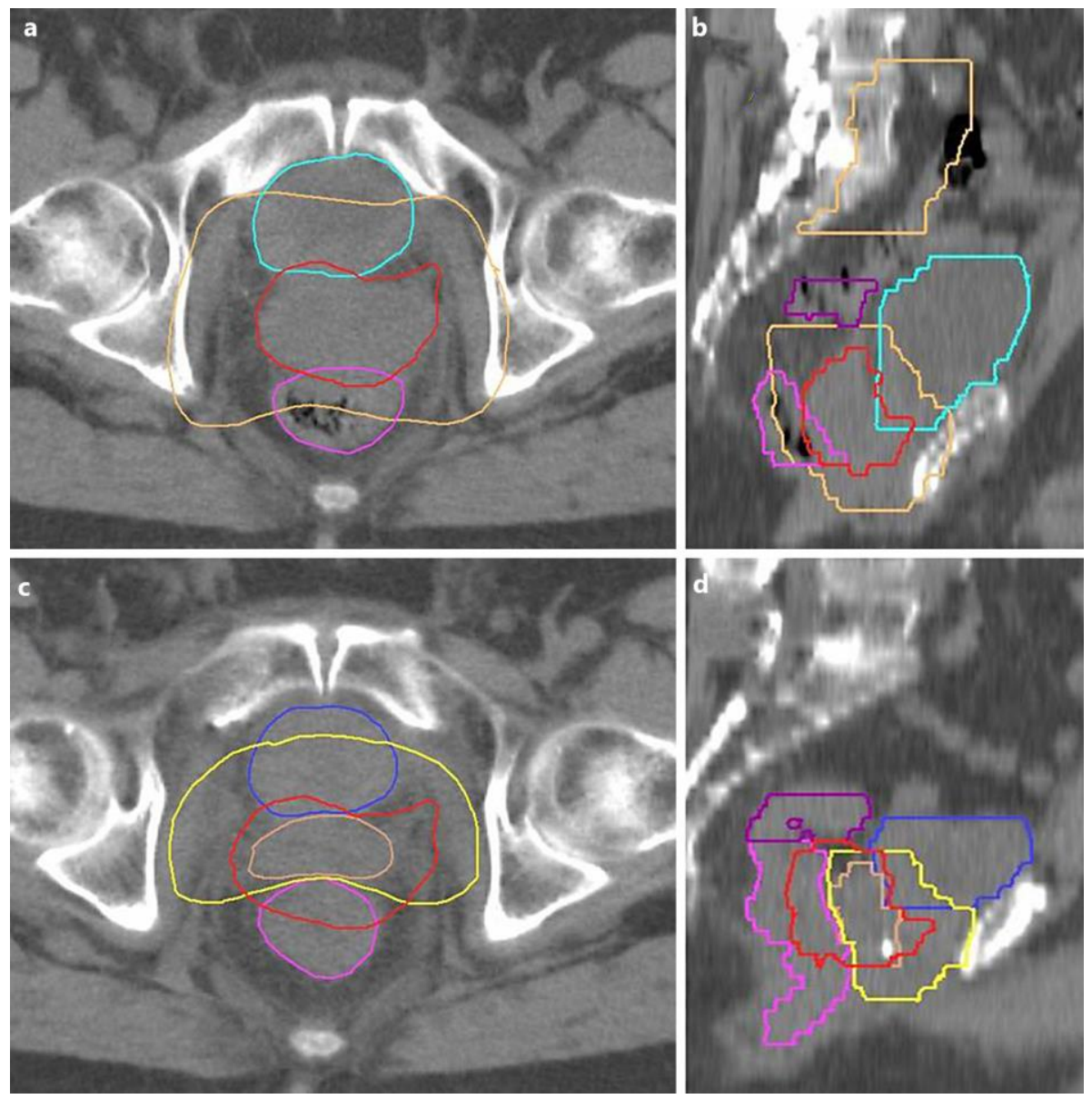

Fig. 2. Reduction in tumor size under radiotherapy. Axial (a) and sagittal (b) planning CT scans before initiation of radiotherapy as well as axial (c) and sagittal (d) planning CT scans after application of 28 fractions (50.4 Gy) are shown. The supposed local recurrence is depicted as a red structure in $\mathbf{a}$ and $\mathbf{b}$ and copied into $\mathbf{c}$ and $\mathbf{d}$. The brown structure in $\mathbf{a}$ and $\mathbf{b}$ represents the target volume (so-called 'planning target volume'), blue the bladder, pink the rectum, and purple the sigmoid. Significant reduction in tumor volume during radiotherapy is seen in $\mathbf{c}$ and $\mathbf{d}$ : the original extension is marked as red, while the actual expansion is marked as brown. The volume of the additional radiation boost is given in yellow. Due to the massive shrinkage of the tumor, the therapy could be completed under avoidance of the rectum. 